\title{
The Level of Public Knowledge about Inflammatory Bowel Disease in Christchurch, New Zealand
}

\author{
Angharad Vernon-Roberts $^{a}$ Richard B. Gearry ${ }^{b}$ Andrew S. Day ${ }^{a}$ \\ aDepartment of Pediatrics, University of Otago (Christchurch), Christchurch, New Zealand; b Department of \\ Medicine, University of Otago (Christchurch), Christchurch, New Zealand
}

\section{Keywords}

Inflammatory bowel disease $\cdot$ Knowledge assessment .

Perceived stigma · Public awareness

\begin{abstract}
Introduction: There is a high degree of perceived stigma among adults with inflammatory bowel disease (IBD), with up to $84 \%$ considering a social stereotype against them due to their condition. This may negatively impact their treatment adherence and quality of life, as well as practical issues such as gaining urgent access to public bathroom facilities. It has been demonstrated that higher public knowledge levels can reduce public stigma, yet little is known about the general level of understanding of IBD in the community. A study was performed to ascertain the public knowledge levels of IBD in Christchurch, New Zealand, using a validated assessment tool. Objectives: The aims of this study were to implement a validated knowledge survey (IBD-KID2) among members of the general public in Christchurch, New Zealand, and to assess the level of understanding about IBD. Methods: Recruitment took place at a Health Research and Education showcase event at the University of Otago (Christchurch), which is a free event open to members of the public. All people over the age of 8 years were invited to participate. Demographic information was collected and the diseasespecific knowledge assessment survey IBD-KID2 completed
\end{abstract}

karger@karger.com www.karger.com/iid

Karger $\frac{1}{\%}$

GOPEN ACCESS
(C) 2020 The Author(s)

Published by S. Karger AG, Basel

This article is licensed under the Creative Commons AttributionNonCommercial-NoDerivatives 4.0 International License (CC BYNC-ND) (http://www.karger.com/Services/OpenAccessLicense). Usage and distribution for commercial purposes as well as any distribution of modified material requires written permission. by all participants consenting to the research. Results: One hundred people consented to the study, and the mean age 39.9 was years (SD 20, range 12-82 years). Of these 100 participants, 71 were female and 71 had a post-secondary education. Twelve participants had a family history of IBD. The mean IBD-KID2 score was 8.7 (SD 2.9), equal to a percentage total score of $58 \%$. No independent variable was found to be significantly associated with IBD-KID2 scores. The frequency of correct answers showed knowledge deficiencies in the areas of nutrition, treatment, growth, and the cause of IBD. Conclusions: This study highlighted knowledge deficiencies among members of the general public in Christchurch, New Zealand. These topics may be addressed with awareness campaigns in order to maximise community support for adults and children with IBD.

(C) 2020 The Author(s)

Published by S. Karger AG, Basel

\section{Introduction}

For many people with inflammatory bowel disease (IBD), their disease is concealable or "invisible" to those around them, and they do not outwardly appear to be sick, which can lead to insensitivity by the public due to a fundamental lack of understanding of the condition [1, $2]$. This poor public knowledge and perception may contribute to a general belittling of IBD symptoms as they 
deal with a less socially acceptable part of the body, which can be a key factor in the daily challenges of someone with $\operatorname{IBD}[2,3]$. Up to $84 \%$ of patients with IBD have perceived stigma, whereby they comprehend a social stereotype to be against them $[4,5]$. The perceived stigma by people with IBD may reduce adherence, self-efficacy, and healthrelated quality of life, as well as increase anxiety and depression $[4,6]$. Public knowledge levels can also have consequences for people with IBD regarding matters such as needing urgent bathroom access in retail and restaurant establishments $[7,8]$.

Previous research has demonstrated that a higher level of public knowledge was the only factor that demonstrated a positive relationship towards decreasing public stigma, such as through media campaigns [5]. Worldwide awareness campaigns such as World IBD Day [9] aim to increase public knowledge, yet understanding still appears to be low and few surveys have been carried out to assess these levels. A national survey conducted in Austria showed that of 1,001 participants, only $31 \%$ had heard of $\mathrm{CD}$ and $20 \%$ of UC, and less than $21 \%$ correctly associated these terms with intestinal disease [3]. In an American survey of 1,200 participants, $11 \%$ had no knowledge of IBD at all and the median rated awareness level was 5.5/10 [5]. In contrast, the public awareness levels of diseases such as diabetes are high [3]. One study was identified that utilized a validated tool to measure knowledge levels of IBD among the general public [10] using the Crohn's and Colitis Knowledge Score (CCKNOW) [11]. Study participants included people with IBD, their friends, and family members, but knowledge levels were still relatively low with a 50\% mean score on the CCKNOW [10].

Besides the benefits of reduced stigma and improved bathroom access, heightened public awareness of IBD will have the crucial impact of promoting early detection and diagnosis that may prevent primary complications from undetected disease progression $[3,12,13]$. The highlighted lack of public understanding of IBD is clearly a fundamental factor in the daily challenges faced by adults and children with IBD, and increasing awareness may result in improved outcomes and help with practical issues related to the disease. No studies have been found that report the public knowledge levels of IBD in New Zealand so as to identify deficiencies in understanding that may be addressed with national awareness campaigns. The objective of this study was to implement a validated IBD-specific knowledge survey, IBD-KID2 [14], among the members of the general public in Christchurch, New Zealand, when they attended a Health Research and Educational showcase event at the University of Otago (Christchurch).
Table 1. Demographic distributions of the study cohort

\begin{tabular}{ll}
\hline $\begin{array}{l}\text { Age, years } \\
\text { Gender }\end{array}$ & $39.9 \pm 20(12-82)$ \\
$\quad$ Male & 29 \\
$\quad$ Female & 71 \\
Level of education* & \\
$\quad$ High school & 29 \\
$\quad$ College & 11 \\
$\quad$ University & 21 \\
$\quad$ Postgraduate & 38 \\
Family history of IBD* & \\
Yes & 12 \\
No & 78 \\
Don't know & 9
\end{tabular}

Values are mean $\pm \mathrm{SD}$ (range) or percentages. IBD, inflammatory bowel disease. * One participant did not provide this information.

\section{Materials and Methods}

\section{Setting and Population}

Recruitment took place at a Health Research and Education showcase event at the University of Otago (Christchurch), New Zealand, where departments from the School of Medicine present displays of their latest research and technology related to their area of speciality. This is a free event open to all members of the public and is advertised through the University Web site, social media, and local high schools. All people over the age of 8 years who were attending the University of Otago (Christchurch) Health Research and Education event in September 2018 were invited to participate in the research. There were no exclusion criteria.

\section{Assessments}

Following the consent process, all participants were asked to provide some basic demographic data. The sole outcome measure of the study was the IBD-KID2 knowledge survey [14]. This survey takes the format of 15 questions: 9 true/false questions and 6 multiple choice. Each question also includes a "do not know" response option (see online suppl. Appendix 1; see www. karger.com/doi/10.1159/000510071 for all online suppl. material). Participant responses are scored as 1 for each correct answer, to a maximum total of 15 . Knowledge domains included general IBD, treatment, and lifestyle/nutrition. IBD-KID2 was selected as this tool has been validated for participants over the age of 8 years and was therefore appropriate for our study cohort inclusion criteria. IBD-KID2 contains items that are not specific to paediatric IBD, whereas adult IBD assessment tools such as the CCKNOW [11] contain items that would not be appropriate for participants aged 8 years and over and have not been validated for children.

Ethics

Ethical approval for the study was provided by the University of Otago Human Ethics Committee (Health) (H16/116). Written, informed consent was obtained from all participants. 
Table 2. The distribution of IBD-KID2 scores between independent variables

\begin{tabular}{llll}
\hline Categorical variables & Group & Mean (SD) & $\begin{array}{l}\text { Significance } \\
(p \text { value })\end{array}$ \\
\hline Gender & Male & $8.8(2.9)$ & 0.89 \\
& Female & $8.7(2.9)$ & \\
\hline Level of education & High school & $7.7(3.2)$ & 0.159 \\
& College & $9.6(2.7)$ & \\
& University & $9.3(2.8)$ & \\
& Postgraduate & $8.9(2.7)$ & \\
\hline Family history of IBD & Yes & $9.8(2.5)$ & 0.17 \\
& No & $8.7(3)$ & \\
& Don't know & $7.3(2.2)$ & \\
\hline Linear variable & Linear regression & & $\begin{array}{l}\text { Significance } \\
(p \text { value })\end{array}$ \\
\hline Age, years & & & 0.301 \\
\hline
\end{tabular}

IBD, inflammatory bowel disease.

\section{Statistical Methods}

Baseline demographics were collected to provide comparisons of baseline IBD-KID2 mean scores against independent variables (age, gender, education level, and personal or family history of IBD). The comparison of mean scores between categorical variables was performed using independent $t$ tests and ANOVA. For continuous variables, significance was tested using linear regression. The significance level was considered as $p \leq 0.05$. Knowledge levels were examined for each item on IBD-KID2 to ascertain areas of good and poor knowledge among study participants. Poor knowledge was considered as less than $50 \%$ of participants answering an item correctly. This figure was selected arbitrarily as while previous studies measuring IBD knowledge have referred to the percentage of correct answers being "good" or "poor," none have provided a rationale for how they classified these categories. It was therefore considered beneficial to have a defined level of acceptable achievement. Statistical analysis was performed using SPSS for Windows [15] and graphs produced using GraphPad Prism version 7.04 for Windows [16].

\section{Results}

\section{Participants}

One hundred attendees agreed to participate in the study, and the demographic distribution of participants examined (Table 1).

\section{IBD-KID2 Scores}

The mean IBD-KID2 score (maximum 15) of all attendees was 8.7 (SD 2.9), representing a percentage total

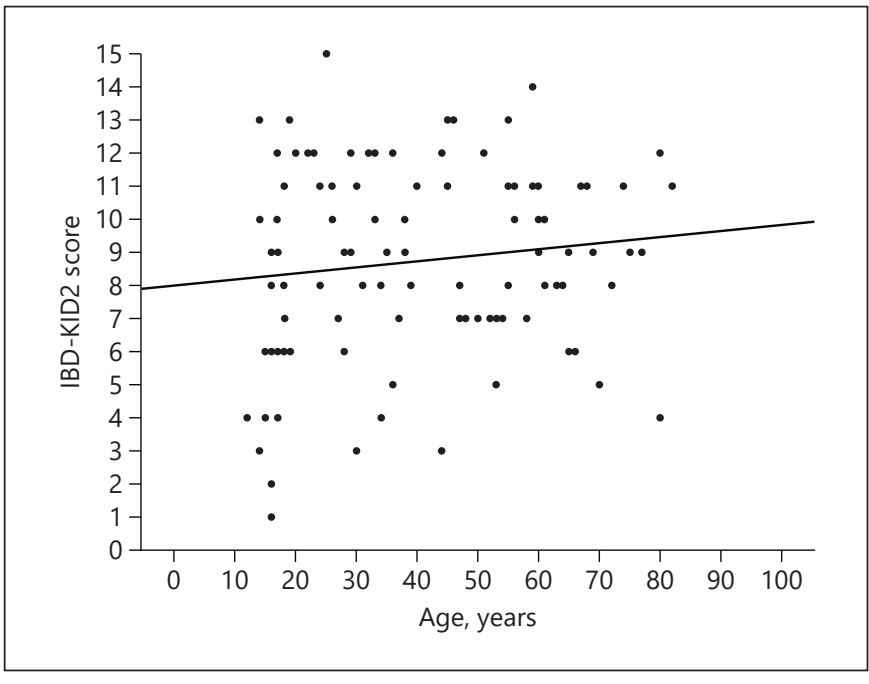

Fig. 1. Association of IBD-KID2 scores against the linear variable of age in years. Line of best fit included.

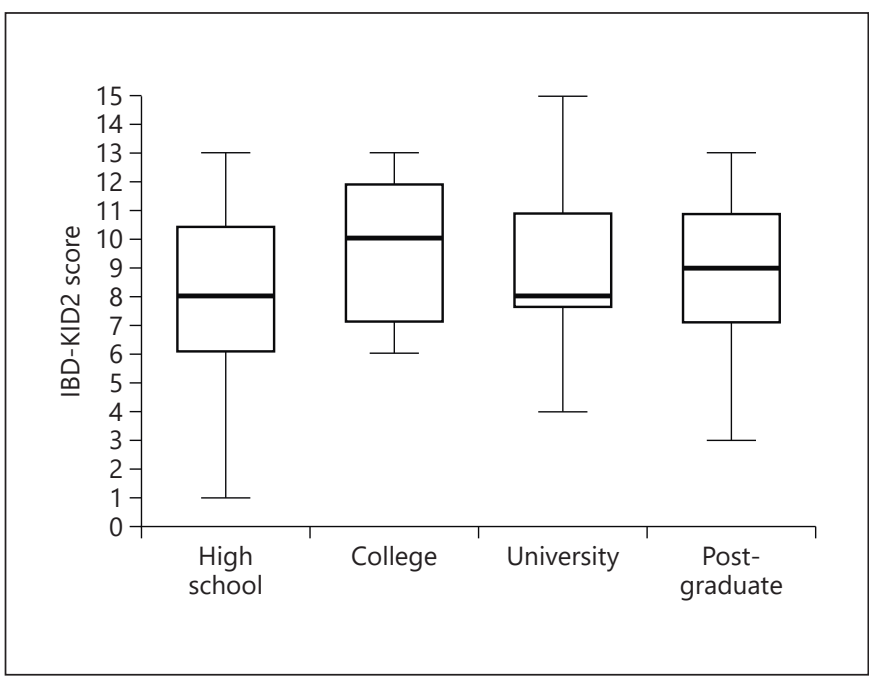

Fig. 2. Participant IBD-KID2 scores for each level of education.

IBD-KID2 score of 58\% (SD 19). When examined against the independent variables, the scores were not significantly different for any groups (Table 2). The 100 participants gave the "Do not know" response 339 times in total (out of 1,500 item responses), representing $23 \%$ of all answers given. When the association between the age of participants and their IBD-KID2 score was explored graphically, it showed great variation (Fig. 1). 
Fig. 3. Frequency of correct answers given to IBD-KID2. The midline represents good knowledge ( $>50 \%$ scoring correctly) or poor knowledge ( $<50 \%$ scoring correctly). CAM, complementary and alternative medicines; EIM, extra-intestinal manifestations.

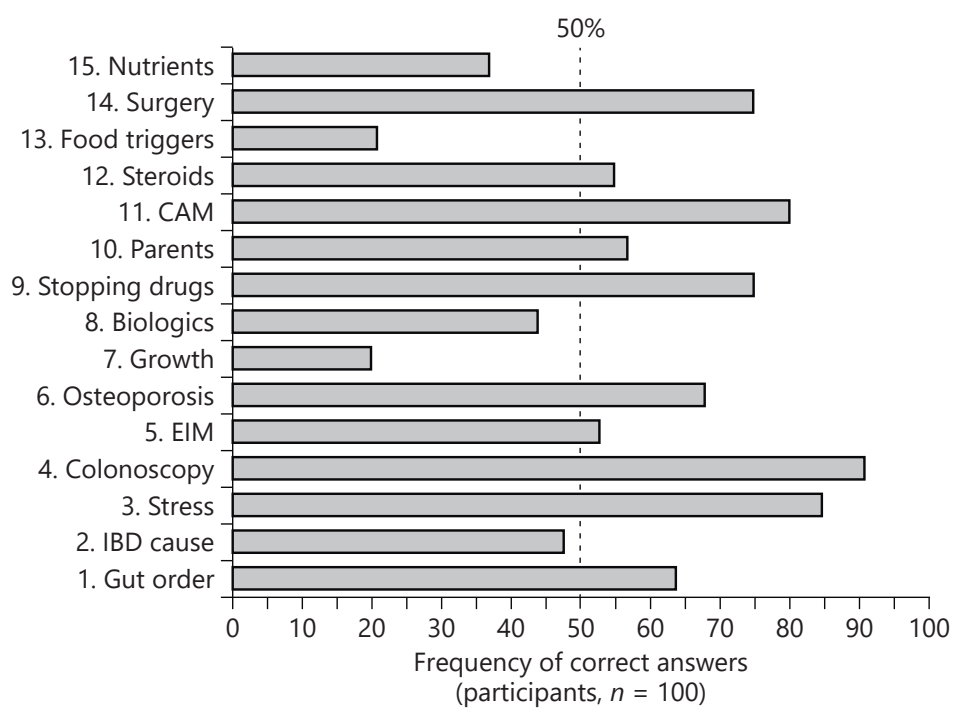

Table 3. Percentage of correct answers for each IBD-KID2 item according to the level of education

\begin{tabular}{lllll}
\hline IBD-KID2 item & $\begin{array}{l}\text { High } \\
\text { school }\end{array}$ & College & University & $\begin{array}{l}\text { Post- } \\
\text { graduate }\end{array}$ \\
\hline 1. Gut order & 52 & 64 & 71 & 68 \\
2. IBD cause & 17 & 46 & 67 & 61 \\
3. Stress & 76 & 82 & 86 & 92 \\
4. Colonoscopy & 86 & 91 & 100 & 90 \\
5. EIM & 62 & 82 & 38 & 47 \\
6. Osteoporosis & 72 & 82 & 57 & 66 \\
7. Growth & 21 & 27 & 24 & 13 \\
8. Biologics & 48 & 36 & 43 & 42 \\
9. Stopping drugs & 66 & 82 & 81 & 76 \\
10. Parents & 59 & 56 & 57 & 58 \\
11. CAM & 62 & 91 & 81 & 90 \\
12. Steroids & 45 & 55 & 68 & 55 \\
13. Food triggers & 7 & 36 & 24 & 24 \\
14. Surgery & 69 & 82 & 76 & 76 \\
15. Nutrients & 31 & 45 & 57 & 29 \\
\hline
\end{tabular}

IBD, inflammatory bowel disease.

When IBD-KID2 scores were plotted against the education level of participants, the results were not significantly different between groups (Fig. 2). Further analysis showed that those with a post-secondary education had significantly higher scores than those with a high school education (mean difference $-1.4, p=0.031$ ). The percentage of correct answers for each individual IBD-KID2 item was examined against the level of education, showing that participants with a high school education level had the lowest percentage of correct answers for 9 items, and the highest scoring for 2 items (Table 3 ).

\section{Knowledge Gaps}

The frequency of correct answers and thus knowledge levels for each IBD-KID2 item were examined (Fig. 3). Ten items (67\%) were answered correctly by more than $50 \%$ of participants.

\section{Discussion/Conclusion}

Little is known about the levels of public knowledge regarding IBD in New Zealand, but the benefits of improved awareness are far reaching. This assessment of knowledge levels among members of the general public in Christchurch has provided a summary of where the general understanding lies and where there are deficiencies that could be addressed that may reduce public stigma and therefore maximize community support for adults and children with IBD.

When comparing the group of participants in this study to others who have completed the knowledge survey IBD-KID2, the mean score overall was at a similar level to that of children with IBD in the original validation study [14], but higher than hospital administrative staff who could also be considered as "general public." While 
the overall level of education did not significantly affect scores in this study, having a post-secondary education was associated with significantly higher scores and in previous studies measuring knowledge specifically in people with IBD, it has also been a positive influence [17-20]. This may indicate that more years of education for people with IBD may motivate people to learn about their own disease and allows a greater understanding of health information, but that this does not necessarily translate to members of the public knowing more about health conditions in general. The lack of association between education level and IBD-KID2 score in this study adds to the validation of construct validity presented in the original validation paper of IBD-KID2 [14] that showed in a group with no expectation of disease knowledge, and questions were answered similarly by those across all levels of education. It may be surmised that this confirms that the knowledge survey IBD-KID2 is simple and easy to read and that the items are measuring knowledge, not reading comprehension, as was considered of the original IBDKID knowledge survey $[19,21]$.

Previous studies among adults with IBD have shown an inverse correlation between knowledge level and age $[17,18,22]$; however, it may not be feasible to make comparisons for such variables as age in a population without IBD as they will not have received disease-specific teaching or education materials. In studies involving adults with IBD, age has been shown to correlate with a deterioration of knowledge levels, possibly due to the fact that people of lower age are engaged in more active social activities and therefore seek more information to better manage their disease [22].

IBD-KID2 does not include items regarding, for example, the cardinal symptoms that may be experienced by people with IBD such as diarrhoea and stool urgency that may be predominant factors that lead to public stigma. However, IBD-KID was developed to be appropriate to the paediatric IBD population and asking questions regarding common symptoms would have increased the questionnaire length with no clear benefit. It should be acknowledged that public knowledge levels regarding factors such as this, in relation to the disease burden of people with IBD, may have greater understanding due to existing public awareness campaigns.

The 5 items of IBD-KID2 that were scored highest $(\geq 75 \%)$ by this study population could be regarded as those most generalizable to other diseases or conditions and therefore less likely to be a source of perceived or actual stigma. The item regarding colonoscopy could be answered correctly by people with knowledge of certain can- cers, coeliac disease, or other gastrointestinal conditions. The use of CAM is widespread for many conditions, so this item could be understood in relation to many other aspects of health care and general well-being. The importance of not stopping drugs may be generalizable to a number of other medication groups, and the effect of stress on the disease may be linked to that of irritable bowel syndrome of which stress is a key factor for triggering symptoms and is a condition with similarities to the cardinal symptoms of IBD that may cause public stigma [23]. The IBD-KID2 items identified as having the lowest correct scores are topics with knowledge deficiencies common among the population of children and adults with IBD, so it would be more unexpected if these items had scored well. These deficiencies highlight areas for public education that may reduce the perceived stigma for adults and children with IBD. One example may be that by increasing the knowledge that poor growth for children with IBD may indicate active disease and therefore explain their greater need for bathroom trips or time away from school that may have been a source of perceived or actual stigma.

\section{Limitations}

It must be acknowledged that the location of recruitment for this study may have introduced selection bias. Members of the public attending the University Open Day may represent a proportion of the local population whose interest in health and education may be greater than average, by virtue of the fact that they attended a university showcase event. In addition, the study population does not represent the general population's education level of New Zealand according to the country's 2013 census [24]. The census gave evidence that the distribution among education levels in New Zealand was as follows: high school $28 \%$, college $52 \%$, university $14 \%$, and postgraduate $6 \%$. Our study population had a comparable number that completed high school as their highest level of education, but higher numbers of those with university and postgraduate education. This may suggest that the study population would be more likely to achieve high scores than those with less interest in health and research; however, the overall level of education made no difference to mean scores. While there was a predominance of female participants, no association was seen between IBD-KID2 scores and gender; however, it is not possible to predict whether similar results would be produced with a more equal distribution between male and female participants.

When comparing variables such as a family history of $\mathrm{IBD}$, a number of factors may influence the strength of 
this effect. When gathering such data, the parameters should be defined as to what constitutes "family history," as demonstrated in previous work that used the term "first degree relative" that found this factor positively influenced knowledge scores $[18,25]$. In this study, as in others [19], participants were asked if a close family member had IBD, without further definition, and neither study found this a significant influence.

\section{Strengths}

Using a validated knowledge assessment tool to measure the level of understanding among members of the New Zealand public has provided insight into where opportunities may lie for improving awareness. The fact that only 1 participant scored $100 \%$ on the tool reflects that the items in IBD-KID2 are not "guessable" for those with higher education levels.

\section{Conclusion}

The IBD-KID2 knowledge assessment tool was developed to be more easily understandable than IBD knowledge surveys such as the CCKNOW [11], which examines the disease and treatments in greater depth. The mean IBD-KID2 score of members of the general public attending the university showcase event was above $50 \%$, thus indicating that knowledge levels were at a level considered to be good. The identified knowledge deficiencies may be targeted in revised public campaigns with the aim of helping adults and children already living with the condition, as well as increasing awareness of the disease that may aid in early detection, thus improving outcomes.

\section{Statement of Ethics}

All procedures performed in these studies were in accordance with the ethical standards of the institutional and research ethics committees where the research was performed and with the 1964 Helsinki Declaration and its later amendments or comparable ethical standards. Ethical approval for the study was provided by the University of Otago Human Ethics Committee (Health) (H16/116). Written informed consent was obtained from all participants.

\section{Conflict of Interest Statement}

Professors Andrew Day and Richard Gearry are on the editorial board of Inflammatory Intestinal Diseases. The authors have no other conflict of interests to declare.

\section{Funding Sources}

Angharad Vernon-Roberts was receiving a doctoral scholarship from the University of Otago when this research was carried out.

\section{Author Contributions}

All named authors made substantial contributions to the conception or design of the work, or the acquisition, analysis, or interpretation of data for the work. All named authors revised the work critically for important intellectual content and gave approval for the final draft to be published. All named authors agree to be accountable for all aspects of the work in ensuring that questions related to the accuracy or integrity of any part of the work are appropriately investigated and resolved.

\section{References}

1 Taft TH, Keefer L. A systematic review of disease-related stigmatization in patients living with inflammatory bowel disease. Clin Exp Gastroenterol. 2016;9:49-58.

2 Bray J, Fernandes A, Nguyen GC, Otley AR, Heatherington J, Stretton J, et al. The challenges of living with inflammatory bowel disease: summary of a summit on patient and healthcare provider perspectives. Can J Gastroenterol Hepatol. 2016;2016:1-5.

3 Angelberger S, Vogelsang H, Novacek G, Miehsler W, Dejaco C, Gangl A, et al. Public awareness of Crohn's disease and ulcerative colitis: a national survey. J Crohns Colitis. 2009;3(3):157-61.
4 Taft TH, Keefer L, Leonhard C, NealonWoods M. Impact of perceived stigma on inflammatory bowel disease patient outcomes. Inflamm Bowel Dis. 2009;15(8):1224-32.

5 Groshek J, Basil M, Guo L, Parker Ward S, Farraye FA, Reich J. Media consumption and creation in attitudes toward and knowledge of inflammatory bowel disease: web-based survey. J Med Internet Res. 2017;19(12):e403.

6 Janicke DM, Gray WN, Kahhan NA, Follansbee Junger KW, Marciel KK, Storch EA, et al. Brief report: the association between peer victimization, prosocial support, and treatment adherence in children and adolescents with inflammatory bowel disease. J Pediatr Psychol. 2009;34(7):769-73.
7 Corinaldi M, Corinaldi M, Dougherty T, Borum ML. Increased education about inflammatory bowel disease can increase support of retail store managers for restroom access legislation. Inflamm Bowel Dis. 2015;21(3):E5.

8 Corinaldi M, Dougherty T, Borum M. P-039 concerns of store managers about employee restroom use are addressed by restroom Access legislation. Inflamm Bowel Dis. 2013;19: S40.

9 World IBD Day. Accessed 2019 Feb 11. Available from: https://worldibdday.org/.

10 Quan H, Present JW, Sutherland LR. Evaluation of educational programs in inflammatory bowel disease. Inflamm Bowel Dis. 2003; 9(6):356-62. 
11 Eaden JA, Abrams K, Mayberry JF. The Crohn's and Colitis knowledge score: a test for measuring patient knowledge in inflammatory bowel disease. Am J Gastroenterol. 1999;94(12):3560-6.

12 Torres J, Petralia F, Sato T, Wang P, Telesco S, Choung RS, et al. 735: disease-specific antibodies and proteomic markers allow for early detection of asymptomatic IBD years before diagnosis: results from the predicts (proteomic evaluation and discovery in an IBD cohort of tri-service subjects) study. Gastroenterology. 2018;154(6, Suppl 1):S152.

13 Colombel J. Targeting the preclinical phase of inflammatory bowel disease. Gastroenterol Hepatol. 2015;11(10):711-3.

14 Vernon-Roberts A, Otley A, Frampton C, Gearry RB, Day AS. Validation of a revised knowledge assessment tool for children with inflammatory bowel disease (IBD-KID2). Inflamm Intestinal Dis. 2020;5(2):70-7.

15 IBM SPSS statistics for windows. $24.0 \mathrm{ed}$. Armonk, NY: IBM Corp; 2016.

16 GraphPad prism. 7.04 ed. La Jolla, CA: GraphPad Software; 2017.
17 Jones SC, Gallacher B, Lobo AJ, Axon AT. A patient knowledge questionnaire in inflammatory bowel disease. J Clin Gastroenterol. 1993;17(1):21-4.

18 Keegan D, McDermott E, Byrne K, Moloney D, Doherty GA, Mulcahy HE. Development, validation and clinical assessment of a short questionnaire to assess disease-related knowledge in inflammatory bowel disease patients. Scand J Gastroenterol. 2013;48(2):183-8.

19 Haaland D, Day AS, Otley A. Development and validation of a pediatric IBD knowledge inventory device: the IBD-KID. J Pediatr Gastroenterol Nutr. 2014;58(3):313-9.

20 Subasinghe D, Wijekoon NS, Nawarathne NM, Samarasekera DN. Disease-related knowledge in inflammatory bowel disease: experience of a tertiary care centre in a developing country in South Asia. Singapore Med J. 2010;51(6):484-9.
21 Vernon-Roberts A, Otley A, Frampton C, Gearry RB, Day AS. Response pattern analysis of IBD-KID: a knowledge assessment tool for children with inflammatory bowel disease. J Paediatrics Child Health. 2020;56(1):155-62.

22 Yoo YS, Cho OH, Cha KS. Disease-related knowledge and information needs among inflammatory bowel disease patients in Korea. Gastroenterol Nurs. 2015;38(6):455-63.

23 Kearney DJ, McDermott K, Martinez M, Simpson TL. Association of participation in a mindfulness programme with bowel symptoms, gastrointestinal symptom-specific anxiety and quality of life. Aliment Pharmacol Ther. 2011;34(3):363-73.

24 New Zealand census stats NZ tatauranga aotearoa; 2013. https://www.stats.govt.nz/infographics/education-and-training-in-newzealand.

25 Day AS, Mylvaganam G, Shalloo N, Clarkson C, Leach ST, Lemberg DA. Assessment of disease-specific knowledge in Australian children with inflammatory bowel disease and their parents. J Paediatr Child Health. 2017; 53(8):778-81. 